\title{
Large-scale screening by the automated Wassermann reaction
}

\author{
W. WAGSTAFF, R. FIRTH, J. R. BOOTH, AND C. C. BOWLEY \\ From the Regional Transfusion Centre, Northfield Road, Crookes, Sheffield
}

SYNOPSIS In view of the drawbacks in the use of the Kahn test for large-scale screening of blooge donors, mainly those of human error through work overload and fatiguability, an attempt was made to adapt an existing automated complement-fixation technique for this purpose. This paper reports the successful results of that adaptation.

Under the Therapeutic Substances Act of 1948, all persons issuing human blood for therapeutic injection are obliged to carry out, on all donations, a screening test for syphilis. The choice of test has been left to the individual directors of regional transfusion centres, and as yet there is little uniformity in the tests employed, these including the Kahn, Berger Kahn, Price precipitation reaction, VDRL slide test, and manual Wassermann reaction (WR) tests. Pugh and Gaze (1965) first reported successful automation of the WR, adapting the Whitechapel technique (Wilkinson, 1962) for use on the standard AutoAnalyzer modules produced by Messrs Technicon Ltd. They reported improved sensitivity and better correlation with existing manual methods when using the Maltaner antigen instead of the crude WR antigen originally employed. This report presents the results of a successful attempt at adapting the method of Pugh and Gaze for the rapid screening of blood donations received at this centre, the automated WR replacing the original one-tube screening Kahn test.

\section{MATERIALS}

COMPLEMENT Freeze dried from Burroughs Wellcome, $2 \mathrm{ml}$ ampoules.

HAEMOLYSIN Rabbit anti-sheep haemolysin (Stayne Laboratories).

SHEEP CELLS These are obtained weekly from a local abbattoir, 300 to $350 \mathrm{ml}$ cells being bled into $240 \mathrm{ml}$ standard acid-citrate dextrose anticoagulant.

Received for publication 31 July 1968.
ANTIGEN Maltaner antigen (Burroughs Wellcome).

BUFFERED SALINE This is made up freshly each week follows:

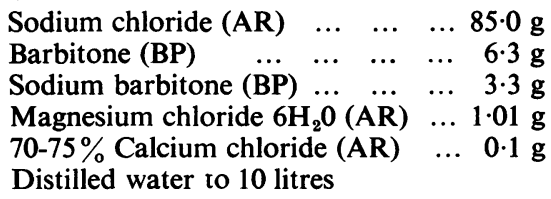

MANIFOLD This has been adapted from the origina Wassermann manifold (Fig. 1): (a) One full doubfe mixing coil has been removed from the complement fixation part of the circuit, ie, between the single capillany mixing coil ' $a$ ' and junction A6 in the water bath $37^{\circ} \mathrm{C}$. (b) One single capillary mixing coil has bein removed from the haemolysis part of the circuit, between junction A6 and the double mixing coil ' $b$ ' in the second half of the circuit at $37^{\circ} \mathrm{C}$. (c) The whole the immersed portion of the circuit has been fused together, eliminating potentially loose glass-to-glass joints made only with Tygon tubing. (d) An antigencomplement-buffer mixture, in the ratio $5: 5: 3$, is pumped into the circuit through a single line, as demonstrated Pugh and Gaze at the Brighton Technicon Symposium (1967).

SAMPLES Serum was obtained from the clotted sample taken with each blood donation for $\mathrm{ABO}$ and Rhests typing. All samples are therefore no more than 48 houtss old when tested, and have been taken under ide्al 'blood donor' conditions. By a fortunate coincidence, the sera are handled by the grouping teams in batches of 40 , and are handed as such to the AutoAnalyeter operator to be transferred to the 40 cups held in eath plate of the Technicon sampler II.

METHODS

COMPLEMENT TITRATION Dilutions of haemolysin ate prepared in buffered saline over a range $1 / 500$ to $1 / 6,000$. 


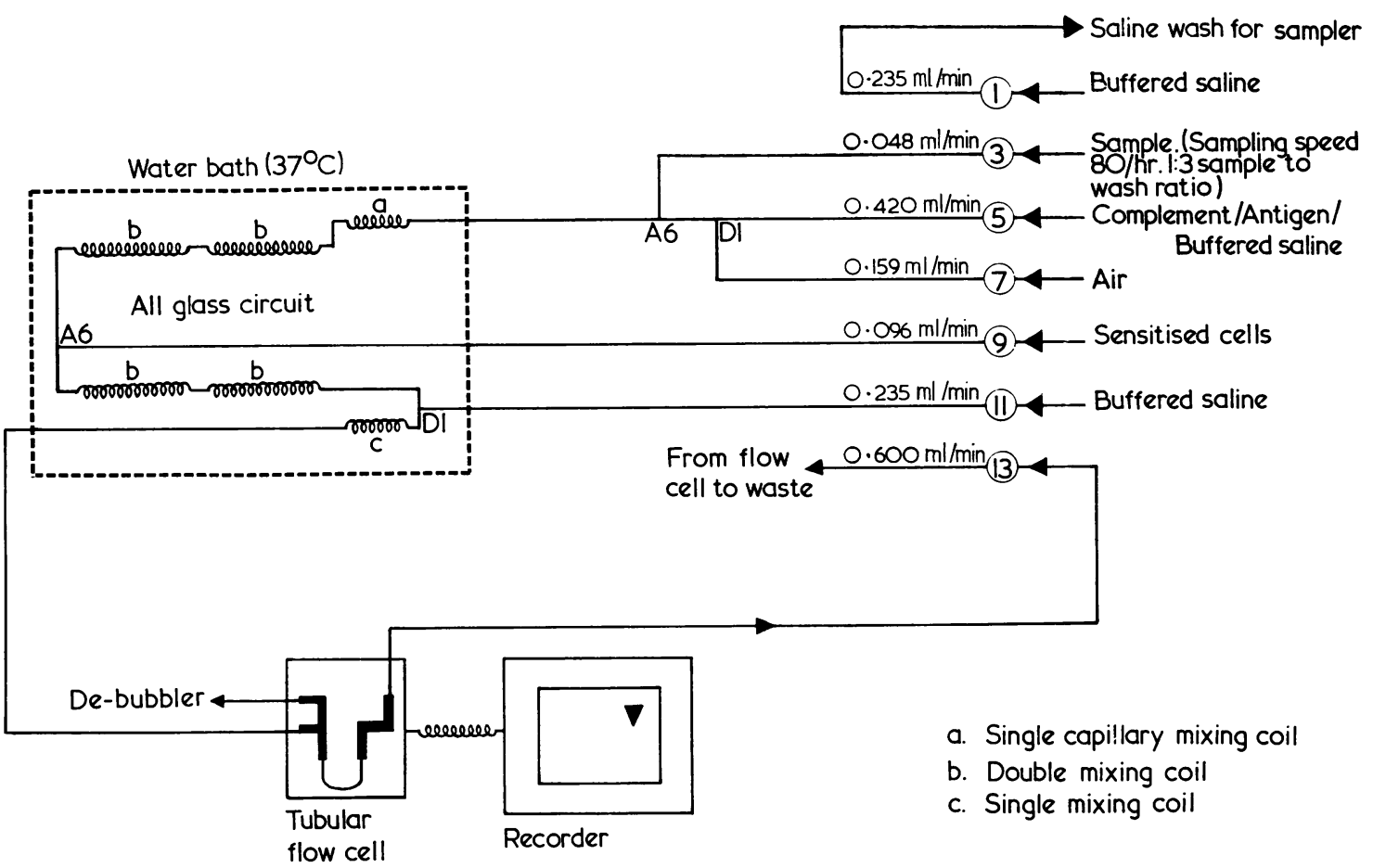

FIG. 1. Test manifold: the DI/A6 junction between pump and water bath has now been replaced by an H3 junction. The tubular flow cell measures $15 \mathrm{~mm}$ and the filters $660 \mu$.

In practice, this range may be shortened when the characteristics of a particular batch of haemolysin are known. Each dilution of haemolysin is used to sensitize an equal volume of $5 \%$ thrice-washed sheep cells. The sensitized cells are then pumped sequentially through the whole circuit, via the sampler and pump tube (size $0.096 \mathrm{ml} / \mathrm{min}$ ) to enter the all-glass circuit at the A6 junction.

Dried complement is reconstituted as directed and dilutions prepared over the range $1 / 80$ to $1 / 140$. Each dilution, mixed with antigen and buffer as above, is pumped via tube 5 , each in turn pumped long enough to cover one full sequence of sensitized cells coming from the sampler.

Buffered saline is pumped via tube 3.

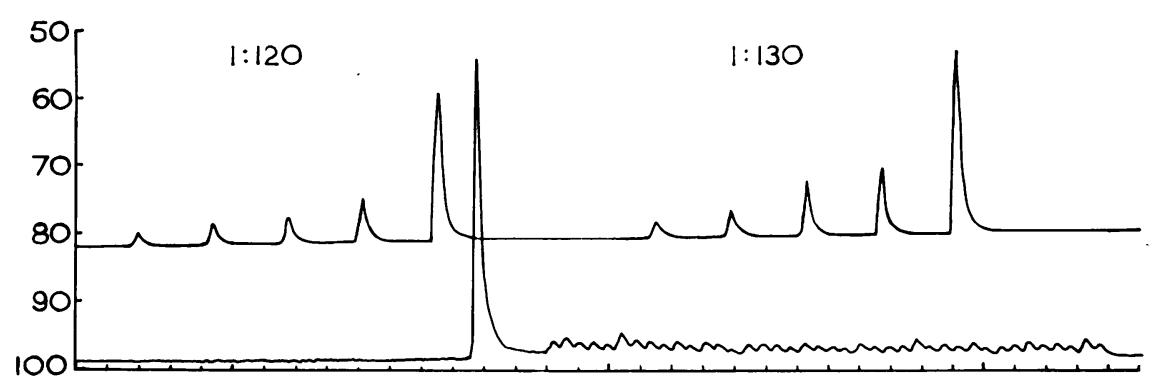

As with all complement haemolysin titration techniques, the combination of the weakest dilution of complement with the weakest dilution of haemolysin which gives complete haemolysis is selected as the basis for calculating the dilution needed in the test proper. These dilutions are conventionally known as the minimum haemolytic doses (MHD) of the reagents. In Fig. 2, the upper tracing depicts part of such a titration, the five sets of cells used being sensitized with $1 / 800$, $1 / 900,1 / 1,000,1 / 2,000$, and $1 / 3,000$ dilutions of haemolysin. From this tracing, the combination of $1 / 120$ complement with $1 / 1,000$ haemolysin was chosen as the MHD of each. The lower tracing shows a test run of one positive control and 40 negative sera, using reagent concentrations calculated from this titration.

FIG. 2. Upper tracing, complement/haemolysin titration. The dilutions of complement are shown, the five aliquots of cells are sensitized with haemolysin at dilutions of $1 / 800$, $1 / 900,1 / 1,000,1 / 2,000$ and $1 / 3,000$. Lower tracing, test run of one positive and 40 negative sera. 
By obtaining supplies of freeze-dried complement in batches, it has been found necessary to titrate only a randomly selected sample from each batch. The use of deep-frozen aliquots of pooled WR-positive sera as a control, and the inclusion of known weakly reacting sera ensure that significant differences in potency between samples of the same batch do not go undetected. With the volume of work handled in this centre, a batch of $180 \times 2 \mathrm{ml}$ ampoules will last for 90 working days. As with conventional manual techniques, only one representative sample from each batch of haemolytic serum need be subjected to titration. Since a batch of haemolysin inevitably lasts longer than one of complement, this means that a complement-haemolysin titration need only be performed with each new batch of complement.

TEST PROPER Sera are separated, after centrifugation, and inactivated, as usual, at $56^{\circ} \mathrm{C}$ for half an hour. Centrifugation is simplified by presentation in racks of 40 precipitin tubes $(50 \times 7 \mathrm{~mm})$ in a Perspex rack designed to fit the Griffin-Christ centrifuge with serological head (Griffin \& George Ltd.) Thus 80 samples can be centrifuged at once.

Reagents are pumped into the system at the rates shown in Fig. 1, using a cam in the sampler II which provides a sampling speed of 80 samples per hour, with a 3:1 wash to sample ratio. Under the conditions described, twin circuits are employed, giving twin tracings on a two-pen recorder, and an actual rate of testing of 160 samples per hour. Each batch of 40 sera is preceded by an aliquot of a pool of WR-positive sera. Complement is pumped at a dilution providing $2 \mathrm{MHD}$, and haemolysin at a dilution providing $1 \frac{1}{3} \mathrm{MHD}$.

From the results of the complement-haemolysin titration shown above, for example, complement would be used at a dilution of 1/60 and haemolysin at a dilution of $1 / 750$.

All samples giving a peak of more than five transmission lines are regarded as suspect and are repeated $(a)$ as a straight repeat with antigen and $(b)$ without antigen, thus providing a control of anticomplementary activity. It is convenient to convert one of the twin circuits for this purpose towards the end of the working day, pumping a complement-buffer mixture through tube 5 instead of the complementary-antigen-buffer mixture. A serum whose peak height in the anticomplementary run is five transmission lines, or more, lower than its peak in the test run, is regarded as positive. Sera with less difference than this are regarded as being anticomplementary.

\section{RESULTS}

Automation of the WR was undertaken specifically, in this instance, to replace the existing manual Kahn test for screening blood donors.

During the period 1 April 1967 to 31 July 1967, 40,435 samples were tested in duplicate. Excluding all cases where results of both Kahn and automated WR tests were negative, the results are shown in Table I. During the period 1 August 1967 to 16 June
TABLE I

RESULTS OF STANDARD TEST (KAHN) BETWEEN 1 APRIL ANF 31 JULY 1967

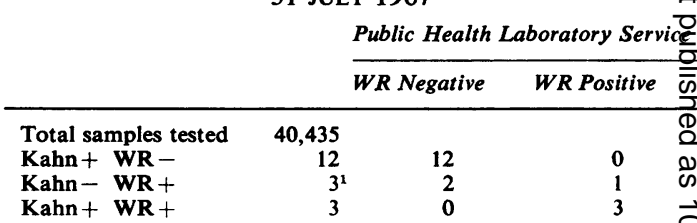
$\left.\begin{array}{l}\text { 1Two cases diagnosed as biological false positive } \\ \text { One case post-vaccinial, negative on repeat }\end{array}\right\}$ All TPI negatize

TABLE II

RESULTS OF STANDARD TEST (AUTOMATED WR) BETWEEN 1 AUGUST 1967 AND 16 JUNE 1968

iv

\begin{tabular}{c} 
Public Health Laboratory Servide \\
\cline { 2 - 3 } WR Negative WR Positive 은
\end{tabular}

\begin{tabular}{|c|c|c|c|}
\hline Total samples tested & 111,942 & & \\
\hline $\begin{array}{l}\text { BTS WR+ } \\
\text { BTS WR- }\end{array}$ & 57 & $42^{1}$ & 15 \\
\hline $\begin{array}{l}\text { Referred because of } \\
\text { previous positive } \\
\text { serology }\end{array}$ & 7 & $7^{2}$ & 0 \\
\hline
\end{tabular}

'Four found WR-negative, Kahn-positive by PHLS, but TPI negati@e. 'Including two donors with repeatedly positive Kahn tests, but TiP negative.

1968, 111,942 samples were tested, using th automated WR as the standard test, the majorify of positive sera also having the Kahn test perform on them (Table II). In all cases, during both periods, sera found positive at this centre were checked oft the Public Health Laboratory, Sheffield, using Maltaner-WR, Kahn, and Reiter's protein comptement-fixation tests. Where appropriate, sera were forwarded to the Central Serological Laboratorg, Manchester, for TPI testing. The results shor clearly that no truly positive sera were missed by the automated technique during the period of duplicated testing. As indicated, the three cases found Kahn negative-WR positive, out of a total of over forty thousand, were diagnosed by the referenge laboratories as being 'acceptable' false positives. During the second phase, a total of $\mathbf{4 2}$ false positives occurred in 111,942 samples tested. Obviously not all sera found negative on the machine could se checked by the reference laboratory during thus time, but 1,230 such randomly selected negatives haFe been checked, all being found negative by methods employed by the Public Health Laboratogy Service.

\section{DISCUSSION}

The original automated technique of Pugh a $\frac{?}{\mathrm{pd}}$ Gaze (1965) has been adapted for screening esseg. tially negative material, the aim always being an 
increase in the rate of testing with maintenance of maximum sensitivity. The sensitivity of the system was increased by cutting the time allowed for haemolysis of sensitized cells to 15 minutes; even so this length of time proves quite adequate for complete haemolysis by the complement remaining after a negative antigen-antibody reaction. As an incidental finding during alterations of this part of the circuit it was discovered that, provided that the sensitized cells are pumped along fine polyethylene tubing and introduced into the circuit via a capillary joint, the capillary mixing coil originally included in the circuit at this point is not really necessary for efficient mixing of the cells. The time allowed for complement fixation was finally fixed at 15 minutes, using the designated coils, complement fixation being apparently complete in this system in 12 minutes. The single capillary mixing coil at the beginning of this phase of the reaction is considered essential.

The fusing of the submerged part of the circuit into a continuous glass unit undoubtedly results in a slightly more elaborate procedure for the complement-haemolysin titration, but this extra trouble is more than compensated for by the reduction in 'noise' on the tracings which results from elimination of so many suspect joints in the system. Regular daily cleaning of the all-glass part of the circuit with chromic acid is also recommended as a noiseeliminating measure. The possibility of cross contamination is also reduced by the same measure, so that at a sampling rate of 80 per hour each individual sample is still easily recognizable, and correlation of any peak on the record with its corresponding sample presents no difficulty in practice. At this sampling rate, loss of sensitivity was obviously to be expected. By incorporating the streamlining measures mentioned a degree of sensitivity is achieved such that a known weakly positive serum (provided as a control by the Public Health Laboratory Service Laboratory) consistently gives a peak of around 20 transmission lines, thereby providing an easy check on sensitivity.

The sera under test occasionally present problems because many donors arrive at a donor session soon after a heavy meal, so that a relatively high proportion of test sera are fatty. Since the measurement within the colorimeter is a nephelometric one of unhaemolysed red cells, the presence of fat globules results in an increase in peak size on the final tracing. This will obviously happen both with and without antigen in the system, so that such sera could be classified as anticomplementary. Prior filtration with a Hemming's bijou bottle filter, using a Ford's asbestos clarifying pad, gives adequate resolution of the problem. Parallel treatment of a positive control serum does not reduce its activity.

Prior mixing of antigen and complement raises the possibility of loss of complement activity during the working day due to anticomplementary activity of the antigen. This has not been found in practice, the repetition of the same positive control before each batch of 40 test sera providing an adequate reagent control.

The results obtained are held to bear out the success of this method in fulfilling our original aim, that of replacing a tedious test with an endpoint open to individual interpretation with an automated test of at least comparable sensitivity, bearing in mind that in screening blood donors for syphilis any error must be towards the false positive rather than the false negative result. To date, no false negative result has been obtained with the machine, either on our own material or on test material kindly supplied by Dr Gillespie. The incidence of false positives given is held to be within acceptable limits. An average working day with the twincircuit machine covers 500 tests, plus repeats, plus maintenance, together with the availability, for part of the day, of a trained technician should he be required for emergency work at other benches. Based on an estimated turnover of around 100,000 tests per year, the cost per test, including machine maintenance, is estimated at one penny more than the Kahn test. Where, however, a higher proportion of positive material is being dealt with, for instance in a Public Health Service reference laboratory, adequate separation of peaks may not be achieved at a pumping rate of 80 samples per hour, and obviously there will be a large number of repeat and anticomplementary tests to be performed. However, the system is felt to be suitable for most laboratories undertaking large scale syphilis testing, providing the mechanics are adjusted to suit the individual requirement.

We gratefully acknowledge the encouragement and guidance given to us by Dr V. W. Pugh and Mr R. W. T. Gaze, of Leicester Royal Infirmary. Our thanks are also due to Dr E. H. Gillespie of the Public Health Laboratory Service, Sheffield, for his continuing help in checking our results and providing us with sera.

\section{REFERENCES}

Pugh, V. W., and Gaze, R. W. T. (1965), Brit.J. vener. Dis., 41, 221.

$\overline{\text { Wilkinson, A. E. (1962). Serological tests for Syphilis. Association of }}$ Clinical Pathologists. Broadsheets No. 41 (New Series). October 1962. 